\title{
VALIDATION OF COOLANT THERMAL RESPONSE IN A TRANSIENT FINITE DIFFERENCE THERMAL TRANSPORT MODEL WITH APPLICATIONS TO FAST SPECTRUM REACTORS
}

\author{
Sydney A. Holdampf ${ }^{1}$, Andrew G. Osborne ${ }^{1}$, and Mark R. Deinert ${ }^{1}$ \\ ${ }^{1}$ The Colorado School of Mines \\ Department of Mechanical Engineering, 1610 Illinois St., Golden, CO, 80401, USA \\ osbornea@mines.edu,mdeinert@mines.edu
}

\begin{abstract}
Transient behavior in nuclear reactors is important in accidents and with reactivity control systems that are driven by thermal feedback. Here, we describe a transient finite difference model for a pin cell system. The fidelity of the model is shown by validation against the thermocouple measurements of the CABRI BI1 experiment and the Safety Analysis SystemSodium Fast Reactor model of the experiment. In the BI1 experiment, a sodium-cooled mixed oxide fuel pin was subject to a loss of flow transient to coolant boiling within a sodium test loop positioned in the center of the CABRI research reactor. Comparisons to the initial steadystate coolant temperature profile, coolant temperature profile at twenty seconds into the transient, and at four axial locations within the coolant show agreement of the simple model with the experimental results better than or similar to those of the Safety Analysis SystemSodium Fast Reactor model. The model can be used to determine the thermal response times of coolant in fast reactors currently operating or in the design phase when subject to loss of flow accidents or other transients. Here, we investigate the difference in coolant thermal response for metal fueled and mixed oxide fueled sodium fast reactors when subject to transient overpower and loss of flow events. Additionally, we determine the effect of pin pitch on outlet coolant temperatures during the overpower event. Finally, we return to the CABRI experiment and show the importance of porosity in fuel temperature calculations.
\end{abstract}

KEYWORDS: thermal response, fast spectrum reactors, transient

\section{INTRODUCTION}

Accurately modeling heat transfer in nuclear reactor cores is central to understanding their safety and performance. While thermal transport directly affects core temperatures, it also affects the stability of the reactor because of feedback effects between fuel and coolant temperatures and neutron balances. Particularly with sodium cooled fast reactors (SFRs), care must be taken to ensure that the coolant reactivity coefficient remains negative over the design parameters. This is typically done by engineering the core to have a high neutron leakage, which increases with coolant temperature. However, high leakage can result in higher refueling costs and less neutron efficiency. Advanced designs have been proposed that rely on devices that inject varying amounts of neutron poison into the core to control its reactivity or that use thermal expansion to do it [1,2]. These passive safety systems are required to ensure negative reactivity feedback to temperature increases in large low leakage reactors, which are able to maintain a high neutron efficiency [3]. The Fast Flux Test Facility demonstrated the success of the Gas Expansion 
Module in an experimental core [4], while the Autonomous Reactivity Control System [5] is designed to operate during the initial phase of the transient where temperatures change rapidly. Due to the time delayed temperature response of the fuel and coolant associated with the thermal inertia of reactor and safety system materials, the thermal response times during transient events become important [6].

Thermal response in light water reactors (LWRs) and SFRs has been investigated in the past by various models. Past studies of thermal response include unprotected loss of flow accidents [7-16], unprotected transient overpower accidents $[8,17,18]$, and unprotected loss of heat sink accidents [15,17]. Several studies have investigated the time to sodium boiling in SFRs or to clad melting in both SFRs and LWRs [7,9,11,19-21]. One study [22] investigates the effect of the pellet-to-cladding gap size on thermal response in a light water reactor. The authors found that the presence of a gap does not significantly impact the cladding temperature but does have a large impact on the fuel temperature profile.

Comparisons of thermal performance of coolant in different types of SFRs were not found. Here, we show the coolant thermal response times of two fast spectrum reactors when subject to a loss of flow and an overpower event. The first SFR is fueled by mixed oxide fuel, and the second contains metal fuel. Prior to our analysis of response times, we begin by confirming the validity of our code by a comparison to experimental and validated model results. Pin pitch is then varied for both types of pins for the same overpower event to determine its effect on outlet coolant temperatures. The effect of porosity on fuel temperature in the CABRI experiment is also investigated.

\section{METHODS}

\subsection{Model Definition}

Figure 1 shows the geometry of the axisymmetric fuel and single phase liquid coolant represented by the subchannel heat transfer model. Modeled geometries include solid or annular fuel pins with a cladding layer, a fuel-cladding gap filled with a gas or liquid, and if present as in a test channel, a structure surrounding the coolant. A hexagonal lattice arrangement is most common in SFRs as seen in the right panel of Fig. 1, but square lattices and cylindrical test channels can be selected using input switches.
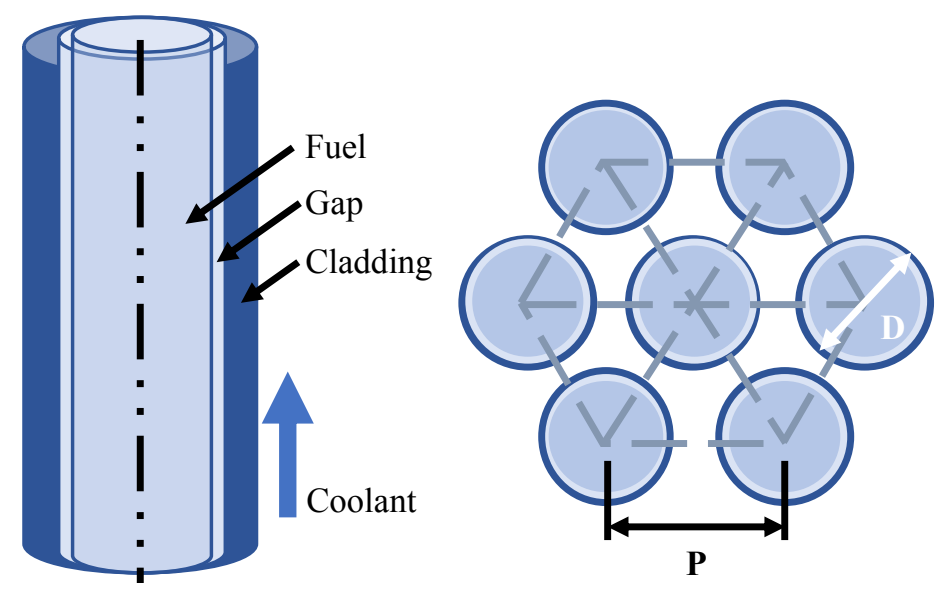

Figure 1. Pin and lattice geometries. Left: axial geometry of the pin-coolant system. Right: lattice geometry. Each triangle is a unit cell in the heat transfer model for a hexagonal lattice, representing half a pin and corresponding quantity of coolant. 
The heat transfer is governed by the transient, radial, one-dimensional heat equation with heat generation coupled to Newton's law of cooling and an open system energy conservation equation, Eqs. (1-3):

$$
\begin{gathered}
\rho c_{p} \frac{\partial T}{\partial t}=\frac{1}{r} \frac{\partial}{\partial r}\left(k r \frac{\partial T}{\partial r}\right)+\dot{q}_{g e n}^{\prime \prime \prime} \\
\frac{\partial q}{\partial t}=-h A\left(T(t)-T_{b}\right) \\
\frac{\partial E}{\partial t}=\dot{m}\left(h_{\text {in }}-h_{\text {out }}\right)+\dot{q}
\end{gathered}
$$

In Eq. (1), $\rho$ is density $\left(\mathrm{kg} / \mathrm{m}^{3}\right), c_{p}$ is specific heat capacity $(\mathrm{J} / \mathrm{kg}-\mathrm{K}), t$ is time $(\mathrm{s}), k$ is thermal conductivity $(\mathrm{W} / \mathrm{m}-\mathrm{K}), T$ is temperature $(\mathrm{K})$, and $\dot{q}_{g e n}^{\prime \prime \prime}$ is volumetric heat generation $\left(\mathrm{W} / \mathrm{m}^{3}\right)$. Heat conduction is assumed to only occur in the radial direction due to the temperature gradient being much greater in the radial direction than in the axial direction [23]. For the convective boundary condition at the cladding edge, Eq. (2), $q$ is the cladding-coolant heat transfer $(\mathrm{J}), h$ is the cladding-coolant heat transfer coefficient $\left(\mathrm{W} / \mathrm{m}^{2}-\mathrm{K}\right), A$ is the cladding outer surface area $\left(\mathrm{m}^{2}\right)$, and $T_{b}$ is the coolant temperature $(\mathrm{K})$. In Eq. (3), $E$ is the energy stored in the coolant $(\mathrm{J}), \dot{m}$ is the coolant mass flow rate $(\mathrm{kg} / \mathrm{s}), h_{i n}, h_{\text {out }}$ are specific enthalpies $(\mathrm{J} / \mathrm{kg})$, and $\dot{q}$ is the heat transfer between the coolant and any surrounding surfaces $(\mathrm{W})$.

Equation (1) is satisfied at all interior locations within the void, fuel, cladding and fuel-cladding gap unless a user defined heat transfer correlation for heat transfer in the gap is provided. In that case Eq. (1) is only satisfied in the void, fuel, and cladding. We assume heat generation only occurs in the fuel.

An energy balance is performed at each boundary node between two materials, Eq. (4) [24]. $U$ is the internal energy $(\mathrm{J}), \dot{q}_{R H S}$ and $\dot{q}_{L H S}$ represent heat transfer into the element from each direction (W), and $\dot{q}_{S}$ is the source or sink term (W).

$$
\frac{d U}{d t}=\rho c_{p} V \frac{\partial T}{\partial t}=\dot{q}_{R H S}+\dot{q}_{L H S}+\dot{q}_{S}
$$

At the outer surface of the coolant channel, an adiabatic boundary condition is applied unless a surrounding structure is present. The axial boundary condition is specified by a fixed temperature at the coolant channel inlet. Equation (2) is the boundary condition at the cladding surface. Equation (3) is discretized axially along the coolant channel. The temperature in the coolant channel is assumed to be radially uniform.

The coupled equations (2-4) are solved using a simple finite-difference approach in space and a CrankNicolson discretization scheme is time. In order to model the coolant channel as a discretized series of open systems, axial coolant nodes are offset by $\Delta z / 2$, half of an axial discretization height, relative to the axial position of the radial nodes.

Thermophysical properties are drawn from literature [25]. Convective heat transfer correlations between the cladding and coolant are obtained from [23,26,27]. Heat transfer in the gap can be modeled assuming solely conduction, using a heat transfer correlation, or with user-specified gap conductances. Properties depend on temperature and are allowed to vary in both space and time throughout the simulation. Initial temperatures and properties are obtained using a thermal resistor model.

The system of coupled equations derived using Eqs. (2-4) can be written as a matrix equation, Eq. (6):

$$
A T=f
$$


Coefficients of unknown temperatures are lumped into matrix, $A$, and the vector, $f$ represents known quantities from the previous time step. Equation (6) was solved at each timestep using the standard Matlab matrix solver library [28].

\subsection{Validation with CABRI BI1 Experiment Results}

During validation, we show that our model can compute accurate transient coolant temperature profiles compared to more complex models. For the evaluation, we compare to the CABRI BI1 experiment, which has also been modeled by ASTEC-Na, RELAP5, SIMMER, CATHARE, and SAS-SFR [19,29,30]. We compare to SAS-SFR as more coolant temperature data points for the CABRI BI1 experiment case are available from the SAS-SFR model. SAS-SFR, the Safety Analysis System-Sodium Fast Reactor code, based on SAS4A developed by Argonne National Laboratory, models subassemblies as single representative pins. SAS-SFR uses the finite difference discretized transient heat conduction equation coupled with an energy equation and point kinetics equations. It accounts for fuel deformation, clad melting, and sodium boiling [19,29-31].

The basis for the BI1 experiment was a RIG-1 MOX pin with a burnup of $\leq 1$ at.\%. The experiment was composed of the pin inside a sodium test loop in the center of a pool-type research reactor. The test loop isolated the experimental pin from the remainder of the core, but the surrounding core allowed normal and accident conditions to be replicated. The BI1 experiment was an unprotected loss of flow event up to sodium boiling [19]. Table 1 provides the geometry of the test pin from the BI1 experiment. To account for the resistance in our model since SAS-SFR used the URGAP model and specified no gap width, gap conductance values were back-computed from steady-state temperature profiles $[19,29,32]$. The asfabricated $\mathrm{O} / \mathrm{M}$ ratio is 1.98 , and the as-fabricated fuel porosity is $6.2 \%$. In our model, we assume the $\mathrm{O} / \mathrm{M}$ ratio increases to 2.0 due to the 1 at.\% burnup and an average porosity of $3.1 \%$ to obtain properties [33].

Table I. Design parameters for the CABRI MOX pin, standard MOX pin, and standard metal pin. $[19,23]$.

\begin{tabular}{cccc}
\hline Pin Type & CABRI BI1 & Mixed Oxide & Metal \\
\hline Fuel Composition & $0.8 \mathrm{U} 0.2 \mathrm{PuO}_{2}$ & $0.72 \mathrm{U} 0.28 \mathrm{PuO}_{2}$ & UPuZr \\
Void and Gap Fill (If Present) & Helium & Helium & Sodium \\
Cladding & Steel & Steel & Steel \\
\hline Inlet coolant temperature $(\mathrm{K})$ & 677.5 & 627.15 & 627.15 \\
Initial coolant mass flow rate $(\mathrm{kg} / \mathrm{s})$ & 0.159 & 0.1786 & 0.1786 \\
Fissile power $(\mathrm{W})$ & 35,738 & 27280 & 27280 \\
Peak power axial location $(\mathrm{cm})$ & 36.18 & 44 & 44 \\
Void radius $(\mathrm{cm})$ & 0.0475 & 0 & 0 \\
Fuel pellet diameter $(\mathrm{cm})$ & 0.6666 & 0.5776 & 0.5776 \\
Cladding thickness $(\mathrm{cm})$ & 0.0505 & 0.04 & 0.04 \\
Pin diameter $(\mathrm{cm})$ & 0.7676 & 0.66 & 0.66 \\
\hline
\end{tabular}

During the transient, the reduction in mass flow rate is governed by Eq. (7). $\dot{m}_{0}$ is the initial mass flow rate $(\mathrm{kg} / \mathrm{s})$ and $t$ is the elapsed time since the initiation of the ULOF.

$$
\dot{m}=\frac{\dot{m}_{0}}{1+\frac{t}{8}}
$$


SAS-SFR and experimental results were drawn from [19] and [29] using ImageJ and the Figure Calibration Plugin [34,35]. The coefficient of determination, $\mathrm{R}^{2}$, shows how well the experimental results fit to our model and the SAS-SFR model, Eq. (8). SSE is the regression sum of squares, and SSTO is the total sum of squares.

$$
R^{2}=1-\frac{S S E}{S S T O}
$$

Two-phase heat transfer is not currently supported by our model. Since sodium boiling in the experiment beings shortly after 20 seconds, simulations are stopped at that point. The axial coolant temperature profile prior to the transient and 20 seconds into the transient are shown in the top panels of Fig. 2. The coefficient of determination, $\mathrm{R}^{2}$, for the fit of the experimental results to our model is 0.9677 and to the SAS-SFR results is 0.9693 prior to the transient. The $\mathrm{R}^{2}$ value after 20 seconds for the fit of the experimental results to our model is 0.9951 and to the SAS-SFR results is 0.9975 .
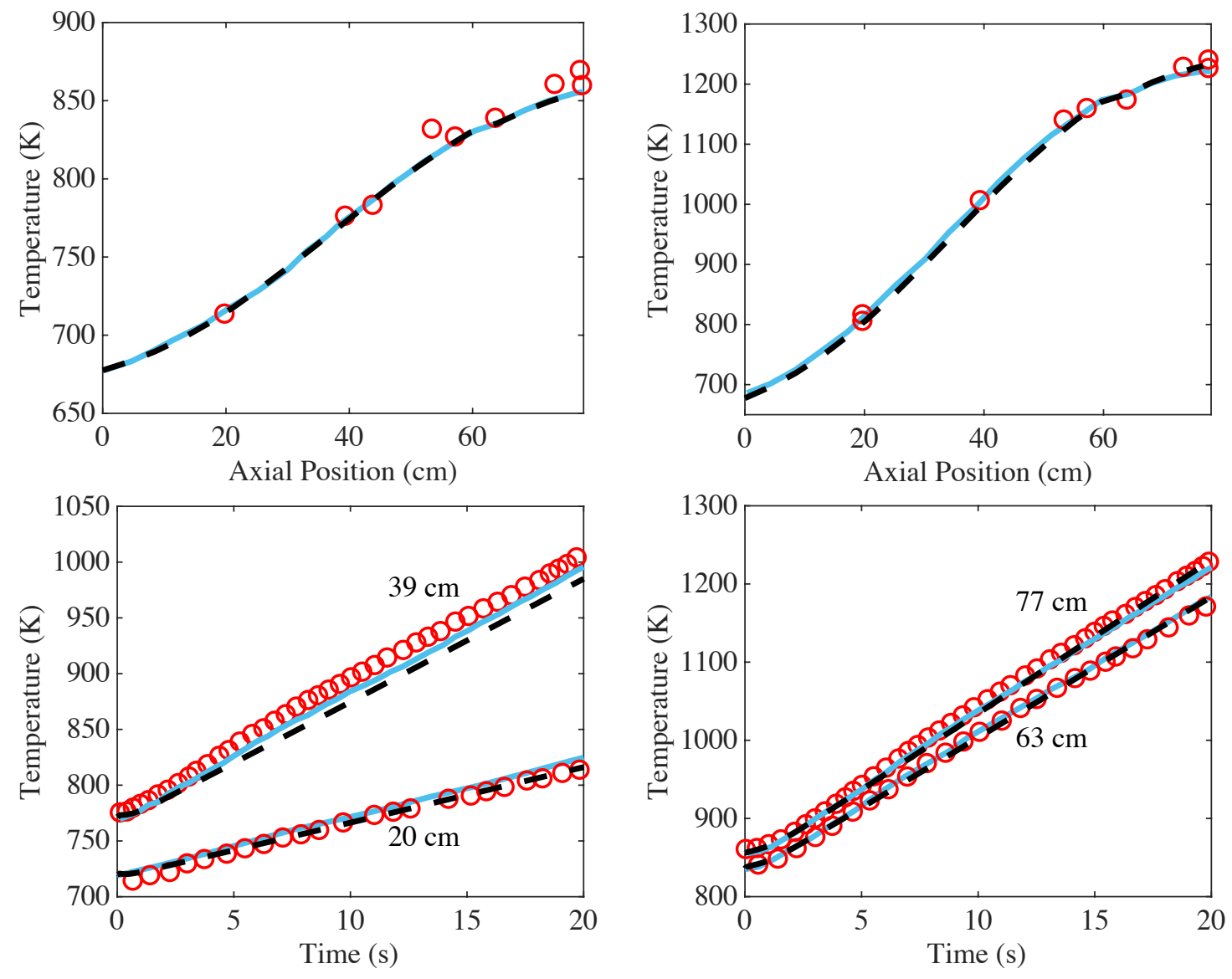

Figure 2. Coolant temperature profiles. (top left) Temperature at steady state prior to the loss of flow event. (top right) Coolant temperature 20 seconds after the loss of flow event begins. (bottom left) Coolant temperature at $20 \mathrm{~cm}$ and $39 \mathrm{~cm}$ axial positions. (bottom right) Coolant temperature at $63 \mathrm{~cm}$ and $77 \mathrm{~cm}$ axial positions. Experimental results shown by red circles in the steady-state data are discrete, in the temporal data they are continuous, SAS-SFR results are shown by the solid blue curve, and our results are shown by the dashed black curve. 
The temperature of the coolant as a function of time was also computed at axial locations 20,39,63 and $77 \mathrm{~cm}$ above the bottom end of the fuel. The bottom left panel of Fig. 2 shows the coolant temperature at the 20 and $39 \mathrm{~cm}$ locations. The bottom right panel of Fig. 2 gives the coolant temperature at $63 \mathrm{~cm}$ and $77 \mathrm{~cm}$ above the bottom of the fuel. The $\mathrm{R}^{2}$ value for the fit of the experimental results to our model at 20 $\mathrm{cm}$ is 0.9942 and to the SAS-SFR result is 0.9573 . The coefficient of determination for the fit to the current model at $39 \mathrm{~cm}$ is 0.9365 and to the SAS-SFR results at $39 \mathrm{~cm}$ is 0.9776 . The coefficient of determination at $63 \mathrm{~cm}$ is 0.9981 for the currents results and 0.9985 for the SAS-SFR results. $\mathrm{R}^{2}$ values for the fit to our current model and SAS-SFR model at $77 \mathrm{~cm}$ are 0.9967 and 0.9964 , respectively. The validation shows this simple approach can be used to model a loss of flow event to a similar accuracy as a more complex model such as SAS-SFR.

\section{RESULTS}

Here we investigate the difference in thermal response time of coolant in mixed oxide and metal fueled fast spectrum reactors during a loss of flow and transient overpower event. The loss of flow transient is a drop in mass flow rate by $20 \%$. The overpower event is an increase in power by $25 \%$. The geometry represented here is characteristic of a BN800 reactor with properties provided in Table I [23]. The metal fuel is $12 \mathrm{a} / \mathrm{o}$ plutonium and $10 \mathrm{w} / \mathrm{o}$ zirconium. The definition of steady state in the models was the time at which the temperature reached $99.9 \%$ of the $\Delta \mathrm{T}$ corresponding to the asymptotic value, which was assumed to be reached in all cases after $\mathrm{t}=10$ seconds.
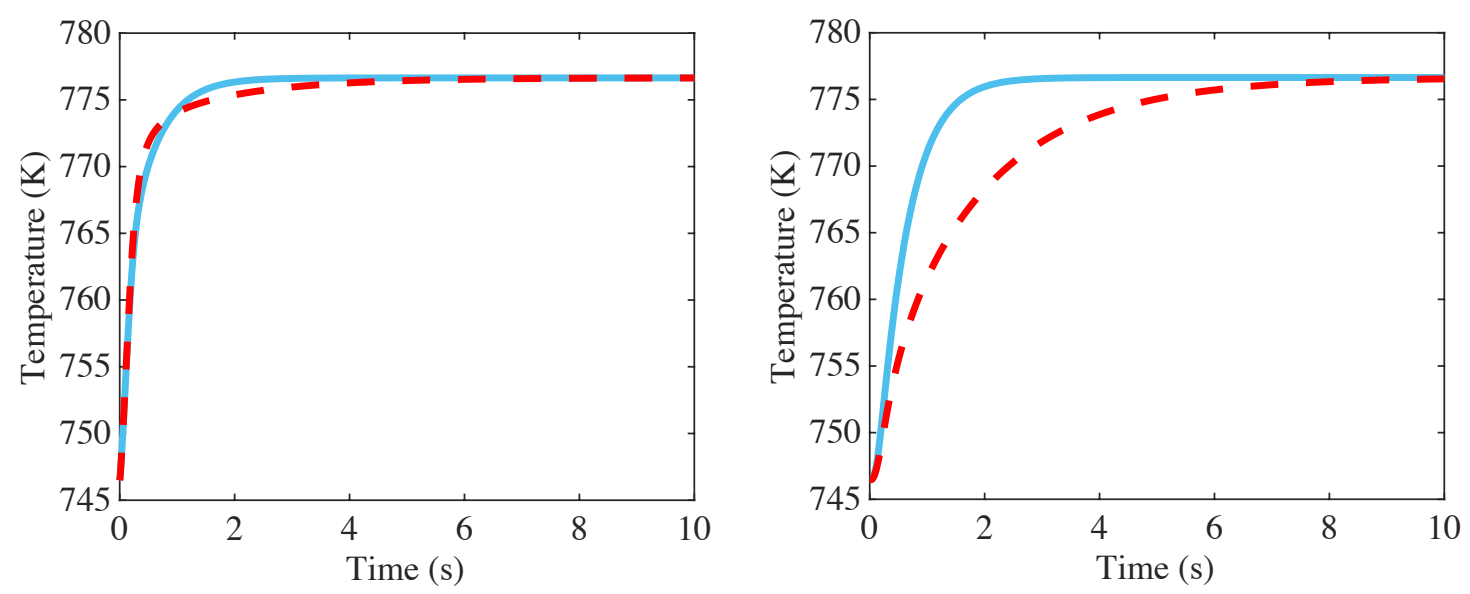

Figure 3. Outlet coolant temperature during the loss of flow event and overpower events. (left) Loss of flow. (right) Overpower. Metal fueled reactor results shown by blue line, MOX fueled reactor results by the red dashed line.

The left panel of Fig. 3 shows that during the loss of flow event, the coolant temperature in the metal and MOX fueled cases reach equilibrium in 3.12 and 7.73 seconds respectively. Figure 3, right, shows that during the overpower event, the coolant temperature in the metal and MOX fueled cases reach steadystate in 3.46 and 9.55 seconds. Additionally, as the ULOF originates in the coolant, both temperature profiles climb more rapidly than when the transient is initiated in the fuel as during the overpower.

Next, we investigate changes in the standard MOX reactor outlet coolant behavior due to pitch size during the same transient overpower event. In our model, pitch variations with the mass flow rate of the coolant held constant do not result in temperature variations since the same amount of heat is being removed. Therefore, the coolant velocity is now held constant instead of the mass flow rate using the same average 
velocity, $6.8146 \mathrm{~m} / \mathrm{s}$, as in the original case. This change can cause slight variations in the temperature results, but as can be seen by comparing the $0.8662 \mathrm{~cm}$ pitch lines of Fig. 4 and the right panel of Fig. 3, the difference is extremely small. It is $0.235 \%$ or less for both fuel types and at all times. A pitch of $0.8662 \mathrm{~cm}$ is the baseline and matches that used in the previous standard MOX versus metal analysis. Figure 4 shows the outlet coolant behavior during the overpower event for the standard pitch $\pm 0.1 \mathrm{~cm}$.

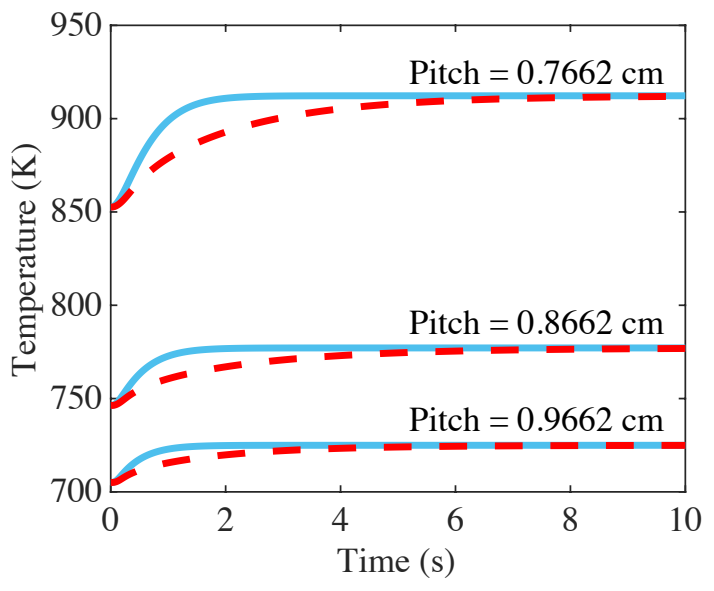

Figure 4. Outlet coolant temperature during an overpower event in MOX and Metal fueled fast reactors with pitches of $0.7662 \mathrm{~cm}, 0.8662 \mathrm{~cm}$, and $0.9662 \mathrm{~cm}$ and constant velocity. Metal fueled reactor results shown by blue line, MOX fueled reactor results by the red dashed line.

As expected, decreasing the pitch increases the initial steady-state outlet temperature due to a smaller body of sodium being available to absorb heat generated by the fuel. Additionally, decreasing the pitch from $0.9662 \mathrm{~cm}$ to $0.7662 \mathrm{~cm}$ increases the temperature increase during the transient from $20.5 \mathrm{~K}$ to 56.7 K.

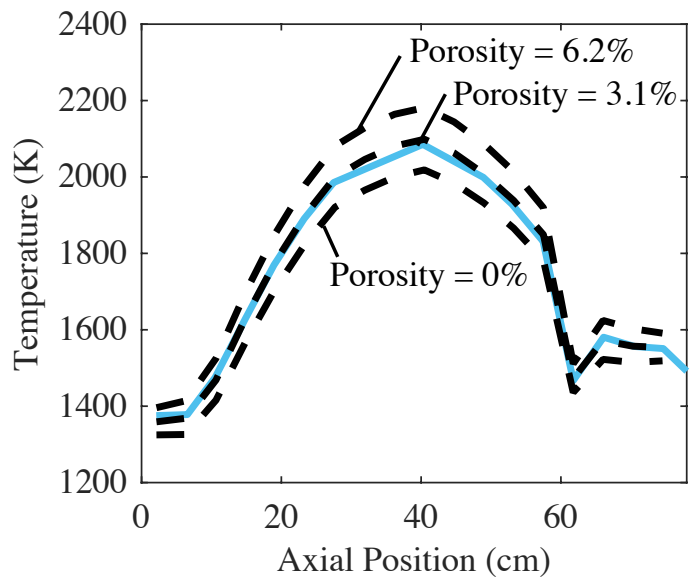

Figure 5. Fuel-void interface temperature prior to the transient during the CABRI BI1 experiment with porosities of $0 \%, 3.1 \%$ and $6.2 \%$ and constant mass flow rate. SAS-SFR results by the solid blue curve, and our results are shown by the dashed black curves.

Next, we return to the CABRI BI1 experiment. Thus far only coolant temperatures have been investigated. Here we look at the fuel temperature profile prior to the transient for three porosity values: no porosity, as fabricated porosity $6.2 \%$, and half the as fabricated porosity $3.1 \%$. Figure 5 shows the difference in our model's results with that of the SAS-SFR model of the experiment. Porosity 
significantly affects the thermal conductivity of the fuel resulting in higher temperatures. From $0 \%$ to $6.2 \%$ porosity, the peak fuel temperature shifts by $163 \mathrm{~K}$.

\section{CONCLUSIONS}

The present work describes a fast, open-source single-channel model for determining the thermal response of coolant in SFRs. It can provide similar results to higher fidelity codes such as SAS-SFR. Our model was used to compute the time behavior of coolant surrounding mixed oxide and metal fueled pins during unprotected transient overpower and loss of flow events. It was found that the temperature of the coolant in a metal fueled pin tends to reach its new equilibrium point much quicker relative to an oxide fueled pin. This would allow a safety system, similar to GEM or ARC described earlier, more time to respond to the overpower events in the mixed oxide fueled reactor. The metal fuel reaching equilibrium quicker is largely due to the higher thermal conductivity of the metal fuel. However, in the unprotected loss of flow event, the coolant in the mixed oxide fueled channel heats up more quickly at first. One possibility is this is due to thermal inertia in the sodium bond.

We analyzed the effects of two different parameters, pitch and porosity. Pitch caused significant changes as expected due to changes in the quantity of water present to absorb heat. As in the initial overpower configuration, the coolant surrounding the metal fuel pin reaches its new equilibrium temperature much quicker than that around the MOX fuel pin for all pitch sizes. Porosity typically is not known and can change during the operating life of a fuel pin, which can cause significant differences when attempting to model actual conditions in pins as seen in the CABRI experiment. In future work, the model will be used to quickly compare thermal response in fast spectrum reactors through principle component analysis or another sensitivity analysis with different geometries, various fuel properties including porosity and $\mathrm{O} / \mathrm{M}$ ratio, and when subject to a variety of accident scenarios.

\section{ACKNOWLEDGMENTS}

This material is based upon work supported by the National Science Foundation Graduate Research Fellowship Program under Grant No. DGE-1646713. Any opinions, findings, and conclusions or recommendations expressed in this material are those of the author(s) and do not necessarily reflect the views of the National Science Foundation.

\section{REFERENCES}

1. C. Pope. Experimental Breeder Reactor II Benchmark Evaluation, Idaho State University (2017).

2. S. Qvist and E. Suvdantsetseg, "Preliminary transient analysis of the Autonomous Reactivity Control system for fast reactors," Prog. Nucl. Energy, 77, pp. 32-47 (2014).

3. E. Suvdantsetseg, S. Qvist, and E. Greenspan, "Preliminary transient analysis of the Autonomous Reactivity Control system for fast reactors," Ann. Nucl. Energy, 77, pp. 47-64 (2015).

4. D.W. Wootan, R.P. Omberg, T. Sofu, and C. Grandy, "Passive Safety Testing at the Fast Flux Test Facility Relevant to New LMR Designs," Proceedings of the IAEA Proceedings of the International Conference on Fast Reactor and Related Fuel Cycles, Yekaterinburg, Russia, June 26-29, 2017 (2017).

5. S.A. Qvist, C. Hellesen, M. Gradecka, A.E. Dubberley, T. Fanning, and E. Greenspan, "Tailoring the response of Autonomous Reactivity Control (ARC) systems," Ann. Nucl. Energy 99, pp. 383-398 (2017).

6. S.A. Qvist, C. Hellesen, R. Thiele, A.E. Dubberley, M. Gradecka, and E. Greenspan, "Autonomous Reactivity Control (ARC) - Principles, geometry and design process," Nucl. Eng. Des. 307, pp. 249274 (2016). 
7. Y. Fukano, "SAS4A analysis on hypothetical total instantaneous flow blockage in SFRs based on inpile experiments," Ann. Nucl. Energy 77, pp. 376-392 (2015).

8. A. Lazaro, M. Schikorr, K. Mikityuk, L. Ammirabile, G. Bandini, G. Darmet, D. Schmitt, P. Dufour, A. Tosello, and E. Gallego, "Code assessment and modelling for Design Basis Accident analysis of the European Sodium Fast Reactor design. Part II: Optimised core and representative transients analysis," Nucl. Eng. Des. 277, pp. 265-276 (2014).

9. H. Ninokata and T. Okano, "Sabena: Subassembly boiling evolution numerical analysis," Nucl. Eng. Des. 120, pp. 349-367 (1990).

10. A.C. Pontedeiro, R.M. Cotta, and J. Su, "Improved lumped model for thermal analysis of high burnup nuclear fuel rods," Prog. Nucl. Energy 50, pp. 767-773 (2008).

11. C. An, F.C. Moreira, and J. Su, "Thermal analysis of the melting process in a nuclear fuel rod," Appl. Therm. Eng. 68, pp. 133-143 (2014).

12. J. Tang, M. Huang, Y. Zhao, S. Maqsood, and X. Ouyang, "Numerical investigations on the melting process of the nuclear fuel rod in RIAs and LOCAs," Int. J. Heat Mass Transf. 124, pp. 990-1002 (2018).

13. S.M. Ghiaasiaan, A.T. Wassel, J.L. Farr, and S.M. Divakaruni. "Heat conduction in nuclear fuel rods," Nucl. Eng. Des. 85, pp. 89-96 (1985).

14. V. Matuzas, L. Ammirabile, L. Cloarec, D. Lemasson, S. Perez-Martin, and A. Ponomarev, "Extension of ASTEC-Na capabilities for simulating reactivity effects in Sodium Cooled Fast Reactor." Ann. Nucl. Energy 119, pp. 440-453 (2018).

15. K. Tuček, J. Carlsson, and H. Wider, "Comparison of sodium and lead-cooled fast reactors regarding reactor physics aspects, severe safety and economical issues," Nucl. Eng. Des. 236, pp. 1589-1598 (2006).

16. C.B. Davis, Evaluation of the Use of Existing RELAP5-3D Models to Represent the Actinide Burner Test Reactor, Idaho National Laboratory, Idaho Falls, Idaho (2007).

17. S. Bortot, E. Suvdantsetseg, and J. Wallenius, "BELLA: a multi-point dynamics code for safetyinformed design of fast reactors," Ann. Nucl. Energy 85, pp. 228-235 (2015).

18. G. Espinosa-Paredes, Espinosa-Martínez, E.-G. Fuel rod model based on Non-Fourier heat conduction equation. Ann. Nucl. Energy 36, pp. 680-693 (2009).

19. A. Flores y Flores, V. Matuzas, S. Perez-Martin, G. Bandini, S. Ederli, L. Ammirabile, and W. Pfrang, "Analysis of ASTEC-Na capabilities for simulating a loss of flow CABRI experiment," Ann. Nucl. Energy 94, pp. 175-188 (2016).

20. M. Bottoni, B. Dorr, C. Homann, and D. Struwe, "State of development of the computer programme BACCHUS-3D/TP for the description of transient two-phase flow conditions in LMFBR fuel pin bundles," Nucl. Eng. Des. 100, pp. 321-349 (1987).

21. J.D. Macdougall, "Lillington, J.N. The SABRE code for fuel rod cluster thermohydraulics," Nucl. Eng. Des. 82, pp. 171-190 (1984).

22. F.A. Soares, R.O. de Castro Guedes, and F. Scofano Neto, "Analysis of the influence of pellet-tocladding gap on the transient heat transfer in nuclear fuel rods via the integral transform technique," J. Braz. Soc. Mech. Sci. Eng. 40 (2018).

23. A.E. Waltar, Todd, D.R.; Tsvetkov, P.V. Fast Spectrum Reactors, 3rd ed., Springer, New York, USA (2012).

24. Nellis and Klein, Heat Transfer; Cambridge University Press, Cambridge, England (2012).

25. Thermophysical Properties of Materials for Nuclear Engineering: A Tutorial and Collection of Data, International Atomic Energy Agency, Vienna, Austria (2008).

26. J. Lamarsh and A. Baratta, Introduction to Nuclear Engineering, 3rd ed., Prentice Hall, New Jersey, USA (2001).

27. W.G. Luscher and K.J. Geelhood, Material Property Correlations: Comparisons between FRAPCON-3.4, FRAPTRAN 1.4, and MATPRO, United States Nuclear Regulatory Commission (2011).

28. Matlab R2018a, The MathWorks, Natick, Massachusetts, USA. 
29. S. Perez-Martin, W. Pfrang, and M.Haselbauer, "Analysis of the CABRI-1 Single Fuel Pin LOF Experiment BI1 with SAS-SFR Code including Two-phase Sodium Behaviour," Proceedings of ICAPP 2014, Charlotte, North Carolina, USA, April 6-9, 2014, pp. 506-514 (2014).

30. G. Bandini, S. Ederli, S. Perez-Martin, M. Haselbauer, W. Pfrang, L.E. Herranz, C. Berna, V. Matuzas, A. Flores y Flores, and N. Girault, "ASTEC-Na code: Thermal-hydraulic model validation and benchmarking with other codes," Ann. Nucl. Energy 119, pp. 427-439 (2018).

31. Argonne National Laboratories Software: SAS4a/SASSYS-1 (Reactor Dynamics and Safety Analysis Code, Argonne National Laboratories Nuclear Engineering Division, Lemont, Illinois, USA.

32. K. Lassmann and F. Hohlefeld. "The revised URGAP model to describe the gap conductance between fuel and cladding," Nucl. Eng. Des. 103, pp. 215-221 (1987).

33. Y. Philipponneau, "Thermal conductivity of (U, Pu)O2-x mixed oxide fuel," J. Nucl. Mater, 188, pp. 194-197 (1992).

34. W.S. Rasband, ImageJ, U.S. National Institutes of Health, Bethesda, Maryland, USA, (1997).

35. F.V. Hessman, Figure Calibration ImageJ Plugin, Institut für Astrophysik, University of Göttingen, Göttingen, Germany (2009). 